\title{
Impact of Solid State Lighting on Energy Utilization and Environmental Conditions
}

\author{
Robert F. Davis \\ Department of Materials Science and Engineering, Carnegie Mellon University, Pittsburgh, PA USA \\ Fax: 1-412-268-3113, e-mail: rfd@andrew.cmu.edu
}

\begin{abstract}
Fuel-based and incandescent lamps convert $\leq 5 \%$ of the energy they consume into visible light; the remaining energy is given off as heat. The conversion efficiency of fluorescent lamps is approximately $20 \%$. These very common sources of light convert the earth's energy sources mostly into waste heat and greenhouse gases. The increasingly precious energy resources and the increasing evidence of pollution-driven climate change demand that we reduce the energy and environmental costs of these forms of artificial lighting both by improving these sources and the employment of new forms of lighting. Light-emitting diodes (LEDs) convert electricity into light using semiconductor materials and, at present, represent the energy efficient technology of the future. Currently, LEDs are primarily used for interior and display lighting, as the efficiency of the diodes "droops" rapidly with an increase in the injected current that is necessary to achieve high-brightness. Exterior solid-state lighting within cities is being tested; however, much research, engineering and technological development remain necessary to achieve increased efficiency, low heat generation and the color temperatures and color rendering that are acceptable to human visual perception.
\end{abstract}

Key words: solid-state lighting, light-emitting diodes, efficiency, energy, environment

\section{INTRODUCTION}

Artificial light has made a significant contribution to the productivity and quality of human life for several generations. It has allowed the expansion of the productive day into the non-sunlit hours of the evening and night and the productive spaces into the non-sunlit (windowless) areas of enclosed dwellings, offices and industrial buildings [1]. Many countries, even continents, could not function in their current manner without artificial lighting; it is so seamlessly integrated into the daily lives of a significant percentage of the human population that they tend not to notice it until the lights go out. However, either large amounts of energy must be generated or substantial quantities of petroleum fuels must be burned to produce this lighting. And all of the widely used techniques to produce light are highly inefficient and in their use result in the generation of enormous quantities of heat and greenhouse gases such as carbon dioxide. Newer, more efficient and much less polluting technologies are now entering the market place of which solid-state lighting (SSL) via the use of light emitting diodes (LED) is the most promising. The following sections detail both the energy and environmental problems and costs associated with lighting as well as the promises and problems currently associates with the realization of SSL in outdoor applications.

\section{COSTS OF CONVENTIONAL LIGHTING}

Because we value artificial light so highly, we consume very large quantities of energy for its production. In the United States, lighting for residential, commercial and industrial use consumes approximately
100 billion kilowatt hours (kWh), 390 billion KWh and 62 billion KWh or about $9 \%, 37 \%$, and $2 \%$, respectively, of the total electrical energy used for each entity. All together the production of artificial light in the United States in 2001 consumed an estimated $22 \%$ of the nation's electricity, equivalent to $8.3 \%$ of the nation's total generated energy [2]. The cost of the energy used for lighting to the American consumer in 2001 was roughly $\$ 50$ billion dollars per year or approximately $\$ 200$ per year for every person in the country. The situation in the European Union (EU) was similar for the initial 15 member countries. For example, in 2006 lighting consumed approximately $12 \%$ of the total amount of electricity used for residential purposes [3]. By comparison, the 10 newer members of the EU used more than $21 \%$ of their residential energy for lighting in that same year; however, they used a smaller average quantity of electricity/household than did the original EU members [3].

From a global perspective, lighting ranks among the end-uses dominating global power demand. Worldwide, grid-based electric lighting consumes $19 \%$ of total global electricity production, slightly more electricity than used by the nations of the Organization for Economic Cooperation and Development Europe for all purposes. Lighting requires as much electricity as is produced by all gas-fired generation and $15 \%$ more than produced by either hydro or nuclear power. The annual cost of this service including energy, lighting equipment and labor is 360 billion US dollars (USD), which is roughly $1 \%$ of global GDP. Electricity accounts for some two-thirds of this US dollar amount [4]. 
A reading of the above paragraphs would likely leave one with the impression that lighting is associated exclusively with electricity. But the reality is different. In fact, about a third of the world's population uses fuelbased lighting. The World Bank estimates the population of people who lack direct access to electric lighting at slightly less than two billion. The extent of rural electrification varies widely from country to country, e.g. about $90 \%$ of the population in Africa is not served by grid electricity, versus $20 \%$ in Mexico. The illumination provided by flame-based lamps is far lower than that achieved from modern electric lighting. The efficiency of fuel-based light production is also very low.

The cost of the energy used for lighting in the US to the environment was approximately 130 million tons of carbon dioxide emitted into the atmosphere or about $7 \%$ of all the carbon emitted by the U. S. in 2001. From a global perspective, the energy consumed to supply lighting entails greenhouse gas emissions on a much more impressive scale: 1,900 million tons of $\mathrm{CO}_{2}$ per year, equivalent to $70 \%$ of the emissions from the world's light passenger vehicles. Fuel-based lighting, used both in vehicles and areas beyond the range of electricity grids, considerably amplifies these consumption figures and the secondary effects of lighting on public health and the environment. At present, 1.6 billion people live without access to electric light, a greater number than when Thomas Edison commercialized the incandescent light bulb in the 1880 s. The paraffin- and diesel-fuelled lighting they use is much less efficient than even the most inefficient incandescent lamp, is a large emitter of $\mathrm{CO}_{2}$ and is very costly. These combined uses provide only $1 \%$ of global lighting but are responsible for $20 \%$ of lighting $\mathrm{CO}_{2}$ emissions. In an era of tight oil markets they consume $3 \%$ of world oil supply - more than the total output of Kuwait.

These aforementioned percentages for energy utilization and $\mathrm{CO}_{2}$ generation are large and, coupled with increasing concern over energy consumption, have inspired the development of new and more energyefficient lighting technologies. In particular, we are currently witnessing a transition from incandescent technology to fluorescent and high-intensity-discharge (HID) technologies - a transition being accelerated in many nations through legislation. In their present forms, however, all of these "traditional" technologies have limitations as noted below. Solid-state lighting is an emerging technology based on LEDs that has made significant progress such that it is now essentially competitive with incandescent technology. The following sections briefly describe the existing lighting technologies and their problems, the fundamental science of SSL, the present status and the future challenges of this newest method of lighting.

\section{ALTERNATIVE LIGHTING SCHEMES}

Filament-based incandescent light bulbs emit light approximately as a blackbody. However, the majority of the emitted radiation lies outside the human visual response. The filaments are typically limited to an operating temperatures in the $2,700-3,200 \mathrm{~K}$ range, and hence to a luminous efficacy of radiation $(\mathrm{lm} / \mathrm{W}$, lumens per watt of radiant energy content of the light, a standard measure of the "visual efficacy" of radiation) of only 16 $\mathrm{lm} / \mathrm{W}$ [2]. The remaining energy from this form of lighting is lost to the ambient as heat.

Glow-discharge-based fluorescent and HID technology depend on the acceleration of free electrons in a gas discharge, the collisions of those energetic electrons with atoms in the discharge, the resulting excitation of those atoms into excited electronic states, and finally the generation of luminescence as those excited electronic states decay. A gas-discharge environment is a complex one, however, and there are many energy-loss channels aside from excitation of atoms into luminescent electronic states [5]. As a consequence, less (and often much less) than $50 \%$ of the injected electrical energy typically ends up in luminescence at the desired wavelengths. Moreover, for mercury-based fluorescent technology, there is an additional energy loss associated with the phosphor conversion of ultraviolet luminescence at $254 \mathrm{~nm}$ to visible luminescence. The luminous efficacies of fluorescent and HID technologies (in an aggregate average over the various lamp types in use in the U.S. in 2001) are about $71 \mathrm{~lm} / \mathrm{W}$ and $96 \mathrm{~lm} / \mathrm{W}$, respectively [4], about $18 \%$ and $24 \%$ that of an optimal multi-component white light source.

The LEDs employed in solid-state lighting are semiconductor diode structures within which DC electrical energy from a battery (or from an $\mathrm{AC}$ electrical outlet converted to DC by a transformer) is converted to a mixture of photons, i.e., light, that appears white to the human eye and that renders the colors of real-world objects through a complex sequence of steps. This sequence of steps can be thought of as "the life cycle of the photon," although, at the point at which the sequence starts (electrical energy in the battery), there is as yet no photon (an analogy to the pupa stage of a butterfly's life cycle might be appropriate). At each stage in the photon life cycle, inefficiencies and parasitic processes cause energy to be lost, so the final amount of energy in the form of photons illuminating real-world objects is a fraction of the initial energy.

Solid-state lighting has the potential to surpass the luminous efficacy limitations of the other forms of lighting and, at the same time, to introduce new functionalities and designs in lighting. The US Department of Energy has in place a large SSL program [6] that extends from fundamental research concerned with the LEDs with enhanced efficiency to development, testing and calibration of LED-based lamps and lighting fixtures for home, workplace and exterior lighting of public places. The goal of this program is to make SSL competitive with fluorescent and HID technologies by 2015 with target external (wall plug) efficiencies in the $35-50 \%$ range, depending on the wavelength of light emitted by the LED. These efficiencies have the potential to enable significant reduction in the rate of world energy consumption. A further benefit is that SSL does not contain toxic materials, whereas the mercury vapor contained in fluorescent lamps is an increasing cause of concern, to the point where used fluorescent lamps must be treated as hazardous waste in many areas. However, SSL is not without its current challenges at 
the levels of scientific understanding, engineering fabrication and technological utilization. The following Section describes the status of SSL via a description of the production and emission of light and the problems associated with each step. Emphasis will be given to the problems currently encountered in LEDs containing $\mathrm{GaN}$ and $\mathrm{InN}$.

\section{SSL - PRODUCT, POTENTIAL AND PROBLEMS}

An inorganic LED (organic LEDs or OLEDs will not be discussed in this document) consists of a chip of semiconducting material treated to create a structure called a p-n (positive-negative) junction, as shown in Fig. 1. The major stages in the photon life cycle include (1) injection and transport of negatively charged electrons and positively charged holes, (2) electron and hole radiative recombination to form a photon within the LED chip, (3) extraction of photons from the LED chip into the surrounding matrix (either encapsulant or air), and (4) mixing of these photons (e.g., blue) with similarly produced photons of other colors (e.g., green and red), or conversion (and mixing) of these photons (e.g., ultraviolet or blue) into photons with longer wavelengths (e.g., blue, green, yellow, red) via the use of photon-stimulated phosphors. The specific wavelength or color emitted by the LED depends on the materials used to make the diode. Red LEDs are based on aluminum gallium arsenide $\left(\mathrm{Al}_{\mathrm{x}} \mathrm{Ga}_{1-\mathrm{x}} \mathrm{As}\right)$ and aluminum gallium indium phosphide $\left(\mathrm{Al}_{\mathrm{x}} \mathrm{Ga}_{1-\mathrm{x}}\right)_{1-\mathrm{y}} \mathrm{In}_{\mathrm{y}} \mathrm{P}$. Blue LEDs are made from indium gallium nitride $\left(\operatorname{In}_{\mathrm{x}} \mathrm{Ga}_{1-\mathrm{x}} \mathrm{N}\right)$ and green from both aluminum gallium phosphide (AlGaP) and $\operatorname{In}_{\mathrm{x}} \mathrm{Ga}_{1-\mathrm{x}} \mathrm{N}$. "White" light is created by combining the light from red, green, and blue (RGB) LEDs or by coating a blue LED with a yellow phosphor.

As indicated above, when the LED is connected to a power source, charge carriers (electrons and electron holes) are "injected" into the negatively doped (n-type) and positively doped (p-type) contact layers of the LED, respectively. Current flows from the p-side or anode to the n-side, or cathode, but not in the reverse direction. The injection takes place through metallic contacts. The injection is not $100 \%$ efficient because contact resistance leads to losses in the process of injecting charge from the metallic contact into the semiconductor. For the technologically important GaN-based materials, this is more of a problem with p-type contacts. These typically have substantially higher resistances than ntype contacts, which can lead to significant energy losses.

The second step is the lateral spread of these electrons and holes to access all portions of the LED's active layer. These semiconductor "current-spreading" layers also need to have very low resistance so that resistive losses do not siphon off a portion of the input energy. Again, for GaN-based materials, p-type spreading layers currently present much more of a problem than n-type, and reducing the resistivities of the former is of considerable concern at this writing. The wider-bandgap $\mathrm{In}_{\mathrm{x}} \mathrm{Ga}_{1-\mathrm{x}} \mathrm{N}$ semiconductors are more difficult to dope than the narrower-bandgap AlGaAs and AlGaInP semiconductors, and as holes are heavier in effective mass than electrons, p-type doping is more difficult in the former materials than n-type doping.

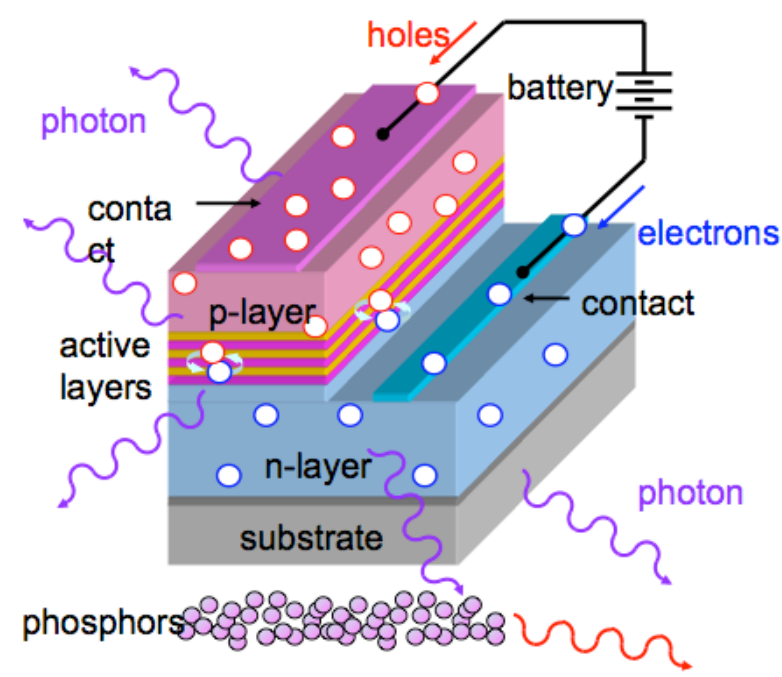

Fig. 1. Schematic of a generic inorganic LED structure illustrating the major stages in the photon life cycle from initial electrical energy from a battery to a mixture of photons that appears white to the human eye and that accurately renders the colors of real-world objects. (From Ref. [6].)

In the next stage in the photon life cycle electrons and holes move into the active layer, typically a series (three or four) of quantum wells, layers of lower band gap semiconductors into which electrons and holes are attracted. Electrons and holes can then combine directly with each other via radiative recombination, with their net energy being given up as a photon or light particle. However, they may also combine with each other via nonradiative recombination. In this case, the electron and hole also annihilate each other, but instead of producing a photon, they produce unwanted heat, in the form of vibrations of the lattice of atoms in the crystal. This energy is wasted and reduces the overall efficiency of the LED. Nonradiative recombination is usually stimulated or initiated by defects in the LED crystal.

The primary cause of high concentrations of extended defects in InGaN LEDs is the lack of readily available and low cost native substrates. InGaN LEDs are currently grown on lattice-mismatched substrates such as sapphire or $\mathrm{SiC}$ and contain threading dislocation (TD) densities of $5 \times 10^{8}-5 \times 10^{9} \mathrm{~cm}^{-2}-4$ to 5 orders of magnitude higher than in typical III-V semiconductors. This apparent insensitivity of InGaN LEDs to TDs is of tremendous benefit for SSL, but understanding of this phenomenon is incomplete. Cathodoluminescence studies of GaN films have provided evidence that TDs are non-radiative defects in nitride materials [7]. More recent calculations have revealed that both the strain field induced by screw dislocations as well as their core configuration can result in preferential localization of one carrier type, causing substantial reduction in electron-hole wavefunction overlap and radiative recombination rates [8]. If TDs are shown to be a roadblock to achieving ultrahigh efficiencies, the growth of InGaN LEDs on more closely lattice-matched substrates may provide a viable solution to dramatically reducing the density of these defects. 
In addition to extended defects, InGaN semiconductors typically contain a range of point defects, including gallium and nitrogen vacancies, and oxygen and carbon impurities. Growth of higher indium compositions requires lower temperatures, which contribute to higher point defect and impurity concentrations [9]. Thus, for green and longer visible wavelength materials, point defects may play a significantly greater role than for shorter wavelength materials. Other point defects produced by interactions include Ga vacancy-defect complexes may also be present.

A second issue related to efficient emission from InGaN materials is the strong polarization effects that arise from their wurtzite crystal structure. In particular, piezoelectric and spontaneous polarization along the [0001] crystal direction leads to large $(>1 \mathrm{MV} / \mathrm{cm})$ electrostatic fields in the multi-quantum well (MQW) active regions of InGaN LEDs (see Fig 1). The primary effect of these fields is to separate electron and hole wavefunctions in the QWs, thereby reducing radiative recombination rates. Because the piezoelectric polarization depends on strain, the reduction in radiative recombination rate is larger for longer emission wavelengths (moving towards the green and yellow) where higher-In-concentration $\mathrm{InGaN}$ is grown on $\mathrm{GaN}$. Mitigation of these polarization effects may be achieved via growth of InGaN QW LEDs on substrates on nonpolar or semi-polar crystal orientations. High quality mplane GaN substrates have now become commercially available. As such, the stage is set for exploring the efficiency limits of InGaN LEDs with nonpolar crystal orientations. However, further advances in non-polar substrate development to achieve low densities of defects over large areas will be required.

A third and very important issue is that at the quantum efficiency of Ga-based LEDs falls as the drive current increases. This detrimental phenomenon is known as droop. Several different explanations have been suggested and, in some cases, reinforced by experimental studies. Investigations at Philips Lumileds Lighting Company have pointed to Auger recombination as the source [10], [11]. By contrast, research at Rensselaer Polytechnic Institute, indicates that polarization fields in the MQW and electron blocking layer enable the escape of electrons from the MQW region and thus are the physical origin of the droop. In other words, droop occurs via carrier recombination outside the MQW region under forward bias. These investigators have shown that through the use of proper quaternary AlGaInN compositions, polarization effects are reduced, thereby minimizing droop and improving efficiency. It has also been suggested that droop is caused by the difficulty of the heavy-mass holes to navigate the MQW structure [13] and by In localization effects with emission occurring in the In-rich regions [14]. In the latter studies, it is suggested that as the local bands fill up when the current is increased, the extra electrons enter the conduction band and move to nearby defects where they recombine non-radiatively and thus diminish the radiative efficiency.

The final problem that will be addressed herein is that there is a range of greenish wavelengths between 540 and $580 \mathrm{~nm}$ where neither InGaN nor InGaAlP have been successfully used to make LEDs with "wall plug"
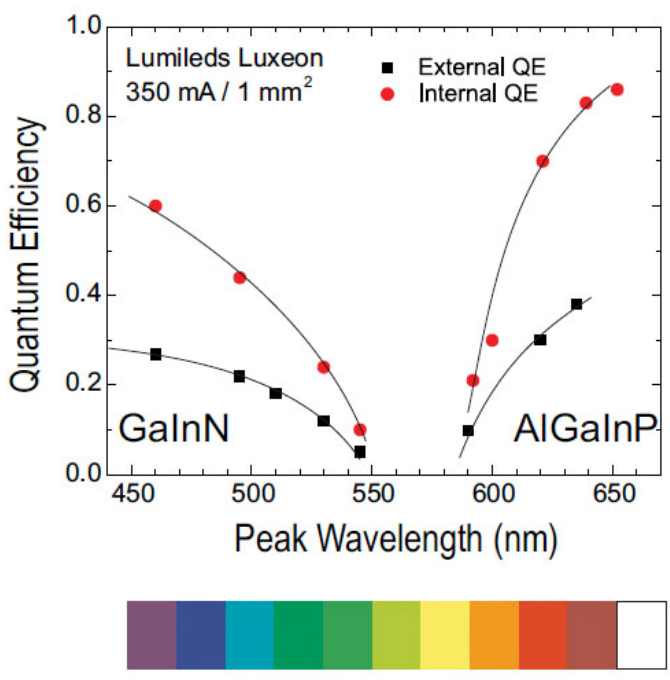

Fig. 2. Plot of external and internal quantum efficiencies vs. wavelength for GaInN and AlGAInP LEDs with different compositions in the active regions. (From Ref. 15.)

efficiencies above $\sim 20 \%$. This green gap is shown in Fig. 2. The major physical changes resulting from the increase in In content needed to make green LEDs, rather than blue ones, are a shift in the lattice constant and a decrease in the bandgap. InGaN degrades as a direct function of In content and temperature. This seriously hampers band gap engineering of the barriers in the active region that is needed to optimize carrier transport and improve strain management. Increasing In content in the QWs also strengthens their internal piezoelectric fields, which turn reduces the electron and hole overlap noted above. None of the above theories for droop can account for the sharp decline in efficiency shown in Fig. 2. Much additional research must be conducted to overcome this persistent problem.

\section{APPLICATIONS OF SOLID-STATE LIGHTING}

The applications of LEDs are ubiquitous, e.g., in driving circuits, power signals, displays, photosynthesis, optical measurements and illumination. One to several millions of these lights can be packaged for use in these applications. However, at present the preponderance of applications require that the viewer look directly at the LED. In this sense, even "high-brightness" or "high efficiency" LED applications are still dominated by indicator lamps. This is NOT solid-state lighting though the term is often used for any lighting that is achieved using any LEDs. Light from a lighting source is viewed in reflection from the illuminated object and the unit of measure is the kilolumen or higher, not lumens (or candela) often used for essentially all the LEDs that one encounters at the present time. Finally, solid-state lighting sources are predominantly white and produce good-to-excellent color rendering. The white light market is emerging in that undercabinet lighting, walkway lighting, task lamps and porch lights have been installed. These lighting fixtures are commonly referred to as "luminaires". However, even here there are problems. Considerable heat is generated due to the inefficiencies currently inherent in the recombination of 
electrons and holes described above. However, the sockets used for incandescent lights were never made to remove heat from this source. Despite some recent engineering to the contrary, just replacing an incandescent or fluorescent light source with an LED source does not make for a high quality and an efficient luminaire. Luminaire manufacturers have found that they must totally reevaluate their approach to the design of any type of fixture to adequately exploit the advantages of LEDs even in rather simple down-light applications. At present, the LED lighting fixture cannot be re-engineered to allow acceptance by incandescent fixtures. As such, those populations that rely heavily on fuel to provide illumination may be in a better position to install LED-base lighting than populations with a significant infrastructure of installed incandescent and fluorescent lighting.

Much larger applications of true solid-state lighting are now beginning to occur. In the United States, streets in. e.g., Ann Arbor, Michigan; Oakland and San Francisco, California; and Chapel Hill, North Carolina as well as parking lots in Portland, Oregon and the main span of a roadway bridge in Minneapolis, Minnesota have been lighted with fixtures containing a large arrays of white-light emitting LEDs. Tests for fatigue, costs and public acceptance are ongoing.

\section{Conclusions}

Lighting at the beginning of the $21^{\text {st }}$ century is inefficient, undemocratically distributed throughout the inhabited areas of the globe, and a major source of pollution either directly from fuel-based lamps or indirectly from the burning of hydrocarbon sources to generate electricity. There has also been dramatic progress in this century in the development of moderately high-power LEDs, especially those that emit in the blue spectrum. However, even these remain an order of magnitude to low in flux per LED and two orders of magnitude to high in cost per lumen to significantly penetrate the general lighting market at this time. Considerable fundamental research, engineering and technology development remains to be conducted. The efficiency of the LEDs must be increased to increase the light output and reduce the heat load, the packaging will need to be continually redesigned and new fixture designs must be implemented to replace the currently installed fixtures that use incandescent and fluorescent lights.

\section{ACKNOWLEDGEMENT}

Financial support for the LED research from which a portion of this article was taken was derived from the US Department of Energy under Contract \#17412.1. 1070083 with Brian Dotson, contract monitor.

\section{REFERENCES}

[1] B. Bowers, Lengthening the Day: A History of Lighting Technology. Oxford University Press, Oxford, England, 1998, p 3.

[2] Navigant Consulting, "U.S. Lighting Market Characterization Volume I: National lighting inventory and energy consumption estimate". U. S. Department of Energy, Office of Energy Efficiency and Renewable Energy, Building Technologies

Program, (2002) pp. $45-50$

(http://www1.eere.energy.gov/buildings/),

[3] P. Bertoldi and B. Atanasiu, "Electricity Consumption and Efficiency Trends in the Enlarged European Union - Status Report 2006" http://sunbird.jrc.it/energyefficiency/pdf/EnEff $\% 20 \mathrm{R}$ eport\%202006.pdf, 2007, p. 38.

[4] IEA, "Light's Labour's Lost: Policies for energyefficient lighting", International Energy Agency, (2006),

[5] J. Boeuf, J. Phys. D, Appl. Phys. 36, R53-79 (2003).

[6] U. S. Department of Energy, Energy Efficiency and Renewable Energy, Solid State Lighting, http://www1.eere.energy.gov/buildings/ssl/

[7] S. J. Rosner, E. C. Carr, M. J. Ludowise, G. Girolami, and H. I. Erikson, Appl. Phys. Lett. 70, 420-422 (1997).

[8] J. You and H. Johnson, J. Appl. Phys. 101, 023516 023518 (2007).

[9] D. D. Koleske, A. E. Wickenden, R. L. Henry, and M. E. Twigg, Journal of Crystal Growth 242, 55-69 (2002).

[10] Y. C. Shen, G. O. Mueller, S. Watanabe, N. F. Gardner, A. Munkholm, and M. R. Krames, Appl. Phys. Lett 91, 141101 - 141103 (2007).

[11] N. F. Gardner, G. O. Müller, Y. C. Shen, G. Chen, S. Watanabe, W. Götz, and M. R. Krames, Appl. Phys. Lett 91, 243506 - 243508 (2007).

[12] Min-Ho. Kim, M. F. Schubert, Q. Dai, J. K. Kim, and E. F. Schubert, Appl. Phys. Lett 91, $183507-$ 183509 (2007)

[13] J. Xie, X. Ni, Q. Fan, R. Shimada, U. Ozgur and J. Morkoc, Appl. Phys. Lett 93, 121107- 121109 (2008).

[14] X. A. Cao, Y. Yang and H. Guo, Jour. Appl. Phys. 104, 093108 - 093114 (2008).

[15] M.R. Krames, O.B. Shchekin, R. Mueller-Mach, G.O. Mueller, Z. Ling, G. Harbers, and M. G. Craford, J. Display Technol. 3, 160-175 (2007).

(Received December 3, 2009; Accepted July 31, 2010) 